\title{
Hydraulically Drained Flows in Rotating Basins. Part I: Method*
}

\author{
Lawrence J. Pratt \\ Woods Hole Oceanographic Institution, Woods Hole, Massachusetts \\ STEFAN G. LLEWELLyN SMith \\ Department of Applied Mathematics and Theoretical Physics, University of Cambridge, Cambridge, United Kingdom
}

(Manuscript received 31 December 1995, in final form 23 July 1996)

\begin{abstract}
An asymptotic method for coupling circulations in basins to hydraulically controlled overflows is introduced. The method is applicable when the forcing, dissipation, and coupling with the overflow are weak, in which case the lowest order solution for the homogeneous or 1/1/2-layer model consists of the natural basin modes including gravity, inertia-gravity, potential vorticity, Helmholtz, and steady geostrophic modes. At the next order of approximation, the mode amplitudes are found to vary slowly with time as the result of forcing, dissipation, interior nonlinear mode interactions, and, most importantly, coupling with the overflow. Even when the latter are absent, the overflow dynamics generally introduce nonlinearity.

Although the basin dynamics are assumed linear to lowest order, the overflow is intrinsically nonlinear. To couple the two systems, the overflow model must be adapted to serve as a nonlinear boundary condition on the basin flow. To do so, a rotating-channel model introduced by Whitehead et al. valid for relatively shallow sills is employed. Although not the central focus, corresponding formulations are derived for straits acting as geostrophic controls or which are dominated by bottom drag.

The principle aim of Part I is to derive the evolution equations governing the coupling between basin and sill. Parts II and III of this work contain a number of examples intended to illustrate the general method and provide insight into physical phenomena associated with hydraulically drained, time-dependent flow in deep basins such as those that occur in the Nordic seas.
\end{abstract}

\section{Introduction}

Evidence of hydraulic control in the Denmark Strait, the Faroe Bank Channel, the Strait of Gibraltar, and a number of other oceanic straits has prompted numerous hydraulic models in the past two decades. ${ }^{1}$ Since these models are intrinsically nonlinear, many simplifications must be made and one of the most far-reaching involves the upstream flow. Typically, it is assumed that the flow originates from a reservoir that is infinite in extent, thereby containing an inexhaustible supply of mass, and in which

\footnotetext{
${ }^{1}$ Reviews can be found in the book of Baines (1995); in Vol. 133, No. 4 (1990), of Pure and Applied Geophysics (R. Hughes, Ed.); in the review of Pratt and Lundberg (1991); and in the book edited by Pratt (1990). Also see recent research articles (Killworth 1994; Dalziel 1991 and references contained therein).

* Woods Hole Oceanographic Institution Contribution Number 8771.

Corresponding author address: Dr. Lawrence J. Pratt, Woods Hole Oceanographic Institution, Woods Hole, MA 02543.

E-mail: 1pratt@whoi.edu
}

the vorticity or potential vorticity is a constant, often zero. For single-layer flows, the product of such a calculation is a relationship between the outflow transport, the sill height or minimum strait width, and the Bernoulli function for the flow (often expressed in terms of the reservoir surface elevation far upstream).

In reality, the circulation in the upstream basin is a product of the forcing and dissipation therein as well as the hydraulic control. Ideally, one would like to calculate the properties of the outflow as functions of the forcing, dissipation, and geometry, and not as a function of the Bernoulli function (which is itself controlled by the forcing, dissipation, and geometry). Furthermore, all oceanic basins contain time-dependent flows, and it is difficult to model this time dependence in a reasonable way when the basin is infinite in extent. For example, observations of deep outflows through the Denmark Strait (Dickson et al. 1990) and Strait of Gibraltar (Bryden and Kinder 1991) show very little seasonal dependence in transport, despite that the processes creating the outflow water are presumably seasonal. It would be difficult to explore this issue using a model with an infinite upstream basin, as forced time dependence might simply radiate far upstream.

There is also a range of interesting scientific issues concerning basin circulations set up by overflows, including 
the general character of the interior circulation and possible boundary currents. Exactly how are overflows fed and what path does the fluid take from the (convective or other) source region to the strait entrance? The effect of the overflow on the normal modes of oscillation and their mutual interactions is relevant to basin energy cascades. The rates of leakage of mass and wave energy from the basin are tied into the issue of seasonal time dependence at the sill.

These considerations suggest the need for exploration of models that couple strait dynamics to forced, dissipative flows in finite basins. In general, such models will be enormously complicated and probably tractable only through numerical means. However, the use of several simplifying assumptions has allowed us to formulate a coupled model that is analytically tractable, or at least amenable to sufficient reduction in complexity that the basic physics can often be illuminated. The purpose is to allow exploration of physical processes that involve coupling between the basin and the strait, which may be difficult to extract from numerical solutions. The method is designed to complement (but not replace) the inevitable numerical model in actual case studies.

The primary assumption used is that the outflows are weak compared to the recirculating transports in the basin interior. Specifically, it is required that the transport velocity (depth-integrated velocity) at the strait entrance is weaker than the typical interior transport velocity by the small factor $\epsilon$. This situation can be arranged by placing a shallow sill or narrow width contraction at some location downstream of the entrance, or through the introduction of strong bottom friction in the strait. The blocking or choking produced by any of these means will reduce the entrance transport velocities as required but still allow for relatively large velocities in the strait interior. The definition of $\epsilon$ will depend on the particular strait model used.

The second approximation is that forcing and dissipation produce effects that are at most $O(\epsilon)$ in the appropriate nondimensional measure. [If forcing and dissipation were allowed to enter the problem at $O(1)$, they would overwhelm the influence of the straits and defeat our attempts to isolate coupled processes.] In addition, we consider basin motions of sufficiently weak amplitude that interior nonlinear interactions arise at $O(\epsilon)$ or smaller. Finally, we assume the fluid to be homogeneous (or with 11/2-layer stratification), though the technique can be extended to multilayer systems.

To the lowest order of approximation, the basins may be considered completely closed and the circulation unforced and linear. The motion can thus be represented as a sum of natural modes, including inertia-gravity, Kelvin, and Rossby-type oscillations as well as a continuum of steady geostrophic modes (provided closed geostrophic contours exist). Some of the properties of the oscillatory modes are reviewed in section 2 . The amplitude $A_{j}$ of each mode is allowed to evolve on a slow timescale $\tau=\epsilon t$ as determined at the next order of approximation, where the outflows come into play along with forcing, dissipation, and possible nonlinear interactions. As described in section 3 , a solvability condition imposed on the $O(\epsilon)$ equations produces a first-order ordinary differential equation for each $A_{j}(\tau)$.

If steady geostrophic motion is possible, the same procedure must be carried out for the associated continuum of modes. The Helmholtz mode, a uniform variation of the surface elevation, is also included in this analysis. Although mass conservation prevents the Helmholtz mode from acting in a closed basin with no sources or sinks, inflows and outflows will generally excite the mode (Miles 1971). It is first necessary to determine the synthesis of Helmholtz and geostrophic modes from arbitrary initial data, a process that has not been done for free-surface (or reduced gravity) flow with general bottom topography. The steps are laid out in section 4 followed by derivation of an integro-differential equation determining the slow time dependence of the geostrophic motion. An alternative form of this equation well suited to numerical algorithms is also derived. For the special case in which the bottom elevation and Coriolis parameter are constant and the oscillatory mode amplitudes $A_{j}$ are all zero, it is shown that $f$-plane quasigeostrophic theory is obtained.

An important point to be made in connection with the analysis is that the outflow conditions and forcing and dissipation, though all weak, determine the eventual distribution of natural mode amplitudes and thus the complete interior circulation. Coupling with the strait acts slowly but is of paramount importance over long timescales. In problems with forcing and dissipation, the effects of the initial conditions generally fade with time and the ultimate solution is determined by the forcing, dissipation, and overflow dynamics.

In order to incorporate the strait dynamics into the theory, the applicable hydraulic model must link the flow at the strait entrance to that at the controlling sill or width contraction. This linkage allows the control to be posed as a boundary condition on the basin flow. Furthermore, it must be shown that constricting the control section reduces the transport velocity at the entrance as measured by the small parameter $\epsilon$. These steps are carried out in section 5 for a strait of rectangular cross section containing a shallow sill. ("Shallow" means that the sill height is nearly as large as the entrance thickness of the outflowing layer.) Under these conditions, the rotating hydraulic model of Whitehead et al. (1974) gives the outflow transport as a nonlinear function of the lowest order surface (or interface) elevation at the entrance. The result takes different forms for various rotation strengths. It is shown that determination of the outflow transport completely specifies the boundary conditions required. If the flow in the strait is not hydraulically controlled, conditions in the basin or sea downstream of the strait can be felt in the upstream basin. In such cases, the throughflow may be blocked or choked by other means, such as strong bottom friction, and the machinery outlined above will remain valid. Although hydraulically controlled outflows remain our main focus, we derive the boundary conditions and $\epsilon$ values for 
several strait models that lack hydraulic control (also section 5).

Some readers may wonder why the normal mode approach is used rather than the Green's function solution common in problems involving oscillations in harbors coupled with open seas (e.g., Miles 1971; Garrett 1975). The first point that should be made in regard to this issue is the necessity of restricting attention to strait flows that are highly choked. If this restriction is relaxed, the equations for the interior flow become nonlinear (as we show in section 5a), and their solutions must satisfy a nonlinear boundary condition at the strait entrance, which precludes analytical treatment, Green's function or otherwise. Tractability generally is available only when the basin is closed to lowest order. Once this approximation is made (and the forcing and dissipation necessarily assumed weak), slowly varying normal modes are the most natural and familiar solution representation. These remarks apply mainly when hydraulic controls are present. Other strait formulations lead to linear problems, including cases where strong frictional effects or linear wave dynamics dominate (as in the harbor problem). Here, forcing and dissipation may be considered finite and Green's function techniques provide a more general solution representation. ${ }^{2}$

Part II (Pratt 1997) of this work is devoted to applications involving steady deep flows in basins fed by various sources, subject to friction in the basin interior, and drained by overflows. Part III concentrates on time-dependent flows. In both studies, comparisons with known features of deep and intermediate flows in the Greenland, Iceland, and Norwegian Seas are made.

\section{Basin modes}

We begin by introducing the governing equations with weak forcing and dissipation and by discussing their lowest order approximations, the linear shallow water equations. We then review several important results regarding normal mode solutions to the latter, including proof that the mode frequencies are real and development of an orthogonality principle. The orthogonality relationship (2.13) is particularly important for later development of the equations coupling the mode amplitues to the overflow dynamics.

Consider a basin of arbitrary shape and undisturbed depth $H(x, y)$ (Fig. 1), connected to other basins or oceans by straits or gaps. Formally, we will consider a geometry with one strait, but the technique is easily extended to include multiple straits as explained later. As suggested in Fig. 1b, the definition of the strait "entrance," the line separating the basin and strait, is arbitrary: the line could plausibly lie at sections A or B. The overriding consid-

\footnotetext{
${ }^{2}$ In practice, further assumptions are often necessary to simplify the Green's integrals to the point where physical intuition can be aided. The most common (e.g., Garrett 1975) is that the strait is vanishingly narrow.
}
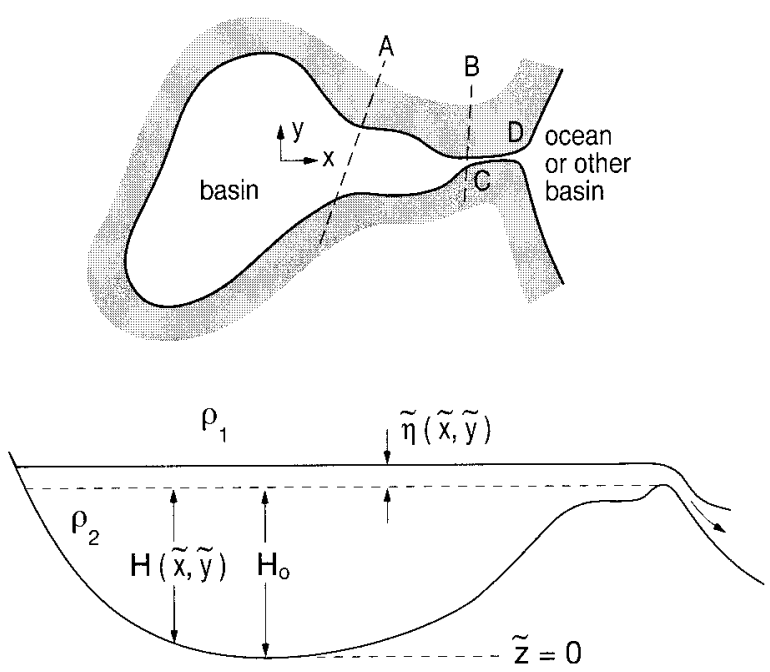

FIG. 1. Definition sketch of basin and strait.

eration is that the strait should be sufficiently narrow that the flow is approximately unidirectional there. We would choose $\mathrm{B}$ rather than $\mathrm{A}$ as the entrance since motions between $\mathrm{B}$ and $\mathrm{D}$ should tend to be more unidirectional than those from A to B.

The deep fluid in the basin is assumed homogeneous. Its upper surface lies at $\tilde{z}=H_{0}+\tilde{\eta}(\tilde{x}, \tilde{y}, \tilde{t})$ and can be considered a free surface or an interface separating an inactive upper layer, in which case the value of gravity $g$ is reduced in proportion to the relative density difference. [Variables which have nondimensional counterparts use ( ) to denote the dimensional version. Table 1 contains a list of notations.] The horizontal velocity components are denoted by $\tilde{u}$ and $\tilde{v}$ and the corresponding transport velocities, $\tilde{u} H$ and $\tilde{v} H$, by $\tilde{U}$ and $\tilde{V}$. The Coriolis parameter $\tilde{f}$ is allowed to vary with $\tilde{y}$.

The starting points for the calculation are the shallowwater equations for the transport velocities

$$
\tilde{\mathbf{U}}_{\tilde{t}}+(\tilde{\mathbf{U}} \cdot \boldsymbol{\nabla})(\tilde{\mathbf{U}} / H)+\tilde{f} \mathbf{k} \times \tilde{\mathbf{U}}=-g H \boldsymbol{\nabla} \tilde{\eta}+\tilde{\mathbf{F}}(\tilde{x}, \tilde{y}, \tilde{t})
$$

$$
\tilde{\eta}_{\tilde{t}}+\nabla \cdot[\tilde{\mathbf{U}}(1+\tilde{\eta} / H)]=\tilde{w}_{p}(\tilde{x}, \tilde{y}, \tilde{t})
$$

where $\mathbf{U}=(U, V), \tilde{\mathbf{F}}$ represents forcing and dissipation, and $\tilde{w}_{p}$ is a positive downward, cross-interface velocity designed to mimic the effects of deep convection or, in the case of a free surface, precipitation minus evaporation.

We now introduce the following nondimensional variables based on timescale $f_{0}^{-1}$, length scale $L_{d}=\left(g H_{0}\right)^{1 / 2}$ $f_{0}$ (the Rossby radius of deformation), surface displacement scale $\mathcal{N}$, and velocity scale $\left(g H_{0}\right)^{1 / 2} \mathcal{N} / H^{0}$ :

$$
\begin{aligned}
(x, y) & =(\tilde{x}, \tilde{y}) f_{0} /\left(g H_{0}\right)^{1 / 2}, & & t=\tilde{t} f_{0} \\
\eta & =\tilde{\eta} / \mathcal{N}, & \mathbf{u} & =\tilde{\mathbf{u}} /\left[\left(g H_{0}\right)^{1 / 2} \mathcal{N} / H_{0}\right] \\
\mathbf{U} & =\mathbf{u} H / H_{0}, & \mathbf{F} & =\tilde{\mathbf{F}} / \epsilon f_{0}\left[\left(g H_{0}\right)^{1 / 2} \mathcal{N}\right] \\
w_{p} & =\tilde{w}_{p} / \epsilon f_{0} \mathcal{N}, & f & =\tilde{f} / f_{0},
\end{aligned}
$$


TABLE 1. List of notations. (All variables dimensionless unless otherwise indicated.)

\begin{tabular}{|c|c|}
\hline Symbol & Description \\
\hline$(\sim)$ & $\begin{array}{l}\text { Dimensional variable. A few other spe- } \\
\text { cially indicated variables without til- } \\
\text { des are also dimensional. }\end{array}$ \\
\hline$x, y, z$ & $\begin{array}{l}\text { Eastward, northward, upward coordi- } \\
\text { nates. }\end{array}$ \\
\hline$H(x, y)$ & Undisturbed dimensional basin depth. \\
\hline$H_{0}$ & $\begin{array}{l}\text { Basin depth scale. Also the maximum } \\
\text { undisturbed basin depth. }\end{array}$ \\
\hline$L_{d}$ & $\left(g H_{0}\right)^{1 / 2} / f_{0}$, the Rossby radius. \\
\hline$\eta(x, y, t)$ & Surface or interface displacement. \\
\hline$N$ & Scale for $\eta(x, y, t)$ \\
\hline$(u, v)$ & Horizontal velocity. \\
\hline $\mathbf{U}=(U, V)=H(u, v)$ & Horizontal tranpsort. \\
\hline $\mathbf{U}_{g}=\left(U_{g}, V_{g}\right)$ and $\eta_{g}$ & $\begin{array}{l}\text { Velocity and displacement for geo- } \\
\text { strophic modes (including the Helm- } \\
\text { holtz mode). }\end{array}$ \\
\hline$\epsilon$ & $\begin{array}{l}\text { Small parameter measuring degree of } \\
\text { constriction of strait. }\end{array}$ \\
\hline Ro & $\begin{array}{l}\text { Rossby number, also }=N / H_{0} \text { by our } \\
\text { scaling. }\end{array}$ \\
\hline()$^{(0)},()^{(1)}$ & $\begin{array}{l}O(0) \text { and } O(\epsilon) \text { terms in asymptotic ex- } \\
\text { pansion. }\end{array}$ \\
\hline$g$ & Gravity or reduced gravity. \\
\hline$\hat{f}(y)=f_{o} f(y)$ & $\begin{array}{l}\text { Dimensional Coriolis parameter. On an } f \\
\text { plane the dimensionless Coriolis pa- } \\
\text { rameter } f \text { is }=1 \text {. }\end{array}$ \\
\hline $\mathbf{F}$ & Momentum forcing vector. \\
\hline$w_{p}$ & $\begin{array}{l}\text { Evaporation, precipitation, or entrain- } \\
\text { ment velocity (positive downward). }\end{array}$ \\
\hline$U_{k}(x, y), V_{k}(x, y), \zeta_{k}(x, y)$ & $\begin{array}{l}\text { Normalized structure functions for basin } \\
\text { mode transport and displacement. }\end{array}$ \\
\hline$\omega_{k}$ & Basin mode frequency. \\
\hline$\tau$ & Slow time $(=\epsilon t)$ \\
\hline$A_{j}(\tau)$ & Basin mode amplitude. \\
\hline\{\}$_{i}$ & See remarks following Eq. (3.7). \\
\hline()$^{*}$ & Complex conjugate. \\
\hline()$_{e}$ & $\begin{array}{l}\text { Denotes variable evaluated at strait en- } \\
\text { trance. }\end{array}$ \\
\hline$W$ & $\begin{array}{l}\text { Strait width (in cases of uniform } \\
\text { width.). }\end{array}$ \\
\hline$W_{s}$ & Strait width at sill. \\
\hline$h_{s}$ & Sill elevation above entrance elevation. \\
\hline$H_{e}$ & Layer thickness at strait entrance. \\
\hline$H_{p}$ & Potential thickness. \\
\hline$H_{s}$ & Mean layer thickness in strait. \\
\hline$\eta_{r}$ & $\begin{array}{l}\text { In WLK theory, } \eta_{r} \text { is the free surface or } \\
\text { interface displacement at right wall } \\
\text { (facing downstream) of strait near en- } \\
\text { trance (see Fig. 2). In the present as- } \\
\text { ymptotic model } \eta_{r} \text { is nearly constant } \\
\text { across the strait entrance. }\end{array}$ \\
\hline$\Delta \eta, \Delta \eta_{\alpha}$ & $\begin{array}{l}\text { Difference in actual and isostatic sur- } \\
\text { face (or interface) level between the } \\
\text { two ends of the strait. }\end{array}$ \\
\hline$U_{s}$ & $\begin{array}{l}\text { Cross-sectional average transport veloci- } \\
\text { ty in strait. }\end{array}$ \\
\hline$L_{s}$ & Length of strait. \\
\hline$R_{f}$ & Linear drag coefficient. \\
\hline n, $\mathbf{l}$ & $\begin{array}{l}\text { Outward unit normal and tangent vector, } \\
\text { either to boundary or to geostrophic } \\
\text { contour. }\end{array}$ \\
\hline $\mathcal{A}$ & Area of basin. \\
\hline C & Geostrophic $\left(f H_{0} / H=\right.$ constant $)$ contour. \\
\hline $\mathcal{A}_{c}$ & Area bounded by $f H_{0} / H$ contour. \\
\hline$C_{e}$ & The contour formed by the basin edge. \\
\hline$\alpha$ & $f H_{0} / H$ \\
\hline
\end{tabular}

where $f_{0}$ is a characteristic value of $\tilde{f}$ and $\epsilon$ is a small parameter measuring the degree of constriction of the strait. In nondimensional terms (2.1) and (2.2) now become

$$
\begin{gathered}
\mathbf{U}_{t}+\operatorname{Ro}(\mathbf{U} \cdot \boldsymbol{\nabla})\left(\mathbf{U} H_{0} / H\right)+f \mathbf{k} \times \mathbf{U}=-\frac{H}{H_{0}} \boldsymbol{\nabla} \eta+\epsilon \mathbf{F} \\
\eta_{t}+\boldsymbol{\nabla} \cdot \mathbf{U}+\operatorname{Ro} \boldsymbol{\nabla} \cdot\left(\eta \mathbf{U} H_{0} / H\right)=\epsilon w_{p},
\end{gathered}
$$

where Ro $=($ velocity scale $) / f_{0}($ length scale $)=\mathcal{N} / H_{0}$ for our scaling. The boundary condition is that $\mathbf{U} \cdot \mathbf{n}$, the normal component of $U$, vanishes along the outer rim of the basin except at the strait entrance, where $\mathbf{U} \cdot \mathbf{n}$ is assumed $O(\epsilon)$.

We now write

$$
\begin{aligned}
& \mathbf{U}=\mathbf{U}^{(0)}+\epsilon \mathbf{U}^{(1)}+\cdots \\
& \eta=\eta^{(0)}+\epsilon \eta^{(1)}+\cdots
\end{aligned}
$$

and, in the subsequent ordering, regard the Rossby number Ro as being $O(\epsilon)$ or smaller. To the lowest order of approximation (2.3) and (2.4) are just the homogeneous shallow water equations

$$
\begin{aligned}
\mathbf{U}_{t}^{(0)}+f \mathbf{k} \times \mathbf{U}^{(0)} & =-\frac{H}{H_{0}} \boldsymbol{\nabla} \eta^{(0)} \\
\eta_{t}^{(0)}+\boldsymbol{\nabla} \cdot \mathbf{U}^{(0)} & =0 .
\end{aligned}
$$

The corresponding boundary condition along the full basin edge is

$$
\mathbf{U}^{(0)} \cdot \mathbf{n}=0 .
$$

The lowest order motion can be described in terms of the usual natural modes:

$$
\left(\begin{array}{l}
U^{(0)} \\
V^{(0)} \\
\eta^{(0)}
\end{array}\right)=\operatorname{Re}\left[\sum_{j=0}^{\infty} A_{j}\left(\begin{array}{l}
U_{j}(x, y) \\
V_{j}(x, y) \\
\zeta_{j}(x, y)
\end{array}\right) e^{i \omega_{j} \mathrm{t}}\right] .
$$

In textbook examples, where the horizontal structure functions $U_{j}, V_{j}$, and $\zeta_{j}$ are separable, the summation is made over two indices.

The issue of orthogonality, which is crucial both to the synthesis of modes from arbitrary initial conditions and to eventual coupling with the strait, can be addressed by writing the equations for the horizontal structure functions:

$$
\begin{aligned}
i \omega_{j} \mathbf{U}_{j}+f \mathbf{k} \times \mathbf{U}_{j} & =-\left(H / H_{0}\right) \boldsymbol{\nabla} \zeta_{j} \\
i \omega_{j} \zeta_{j}+\boldsymbol{\nabla} \cdot \mathbf{U}_{j} & =0
\end{aligned}
$$

along with the complex conjugate forms

$$
\begin{aligned}
-i \omega_{k}^{*} \mathbf{U}_{k}^{*}+f \mathbf{k} \times \mathbf{U}_{k}^{*} & =-\left(H / H_{0}\right) \nabla \zeta_{k}^{*} \\
-i \omega_{k}^{*} \mathbf{U}_{k}^{*}+\nabla \cdot \mathbf{U}_{k}^{*} & =0 .
\end{aligned}
$$

Taking $\left(\mathbf{U}_{k}^{*} H_{0} / H\right) \cdot(2.9)+\left(\mathbf{U}_{j} H_{0} / H\right) \cdot(2.11)+\zeta_{k}^{*}(2.10)+$ 
$\zeta_{j}(2.13)$, integrating the result over the area $\mathcal{A}$ of the basin, and applying the boundary condition (2.7) leads to

$\left(\omega_{j}-\omega_{k}^{*}\right) \iint_{\mathcal{A}}\left[\zeta_{j} \zeta_{k}^{*}+\left(H_{0} / H\right) \mathbf{U}_{j} \cdot \mathbf{U}_{k}^{*}\right] d \sigma=0$.

If $k=j$, then $\omega_{k}=\omega_{k}^{*}$ and the frequencies are therefore real. If $k \neq j$ and $\omega_{k} \neq \omega_{j}$ then (2.13) gives an orthogonality condition in terms of area integration. However, if frequency multiplicity exists $\left(\omega_{k}=\omega_{j}\right.$ for $\left.j \neq k\right)$, it is still possible to construct orthogonal eigenfunctions corresponding to each degenerate root, a consequence of the self-adjoint nature of the system (Platzman 1972). Other properties of the basin modes, including distribution of frequencies, were originally analyzed by Veltkamp (1960) and are summarized by Platzman (1972). In particular, it can be shown that for $f$-plane motion $(f=1)$ with uniform depth $H=H_{0}$, superinertial $(|\omega|>|1|)$ frequencies are discrete and infinite in number. Multiple modes sharing the same frequency may exist, but the multiplicity is finite. In the subinertial $(|\omega| \leq|1|)$ range the spectrum is discrete and contains a finite number of modes, each having finite multiplicity. The sole exception is the root $\omega=0$, corresponding to steady geostrophic motion, for which a continuous distribution of modes may exist. When $H$ or $f$ is variable, the number of subinertial modes becomes infinite, either through an infinite number of discrete frequencies or through a certain frequency with infinite multiplicity or both.

The orthogonality relation (2.13) can be used to synthesize any oscillatory $\left(\omega_{j} \neq 0\right)$ mode from arbitrary initial data. Steady "modes" $\left(\omega_{j}=0\right)$ will also generally exist and additional conditions are required for their synthesis. The simplest mode is a uniform displacement of the free surface or interface with no associated horizontal motion and is often referred to as the Helmholtz mode. Although it can be removed in single-basin problems through redefinition of the reference elevation, the mode is nontrivially present in coupled problems. If the basin contains some closed "geostrophic" contours (along which $f H_{0} / H$ is constant), a continuum of steady geostrophic solutions is also possible. According to (2.8) the interface displacement due to these modes and the Helmholtz mode is $\mathcal{A}_{0} \xi_{0}$ where $\mathcal{A}_{0}$ is constant. To simplify the notation somewhat, we represent $\mathcal{A}_{0} \xi_{0}$ by $\eta_{g}$ and, likewise, set $\mathcal{A}_{0} U_{0}=U_{g}$ and $\mathcal{A}_{0} V_{0}=V_{g}$. The procedure for synthesis of these modes from arbitrary initial data is discussed by Greenspan (1969) for circulation in a container with a rigid lid. The present free-surface (or interface) problem, which is a bit more involved, is taken up in section 4.

\section{The alteration of basin modes by a strait}

This and the next section contain the key results, namely, the relationships governing the slow time evolution of the normal mode amplitudes. In this section we develop the evolution equations for the oscillatory $\left(\omega_{k} \neq 0\right)$ modes. It is possible for the reader to skip the technical details and proceed directly to the result [Eq. (3.5)] and its interpretation, immediately following, as an energy equation. For each mode amplitude, there is a first-order (in the slow time variable) differential equation containing forcing terms associated with the outflow, the explicit forcing and dissipation, and nonlinear interactions with other modes. In order to evaluate these forcing terms, one must use information about the outflow boundary condition (section 5). It is also possible that one must simultaneously compute the evolution of the modes for which $\omega_{k}=0$, and this is discussed in section 4.

At the $O(\epsilon)$ approximation of the expansion introduced in the previous section, the effects of the strait, the forcing and dissipation, and nonlinear mode interactions come into play. Though weak, these influences ultimately determine the distribution of mode amplitudes (and therefore the total solution) for the particular problem at hand. In accordance with the usual outcome of perturbation techniques we anticipate that the weak forcing, dissipation, and nonlinearity will produce secular growth in time of the $O(\epsilon)$ solutions, an effect we will attempt to remove by introducing a slow time dependence in the $O(1)$ solutions. Formally, we let $\mathcal{A}_{j}=\mathcal{A}_{j}(\tau)$, where $\tau=\epsilon t$, and use this slow time dependence to remove any secular terms. The compatibility condition determining $\mathcal{A}_{j}(\tau)$ is developed in this section for the oscillatory modes and in section 4 for the geostrophic modes. In many problems most of the desired information concerning the physics of the coupled processes will be contained in the evolution equations for $\mathcal{A}_{j}(\tau)$, and it will not be important to explicitly solve for the $O(\epsilon)$ fields. This is true in problems involving forced steady circulations with inflows and outflows, unforced geostrophic adjustment, problems involving time-dependent forcing over broad frequency bands, or forcing at resonant or nearresonant $^{3}$ frequencies. In the subinertial range where natural frequencies are densely packed, it will often be the case that any forcing frequency is resonant or near-resonant. If the secular terms are absent (as in the case of nonresonant forcing) and the initial state motionless, the entire $O(1)$ solution is zero and the perturbation approach may provide no inherent simplification. In some cases, motions may arise with amplitudes involving fractional powers of $\epsilon$, and these require special treatment. One example is the "arrested topographic wave" of Csanady (1978), which is discussed in Part II. Even when the $O(1)$ solution is nonzero, some analysis of the $O(\epsilon)$ fields may be necessary in order to gain insights into Lagrangian aspects of the flow field or to compute western boundary currents (also see Part II).

To $O(\epsilon)$, Eqs. (2.3) and (2.4) are

\footnotetext{
${ }^{3}$ Near resonance means that the forcing frequency minus the nearest natural frequency is $O(\epsilon)$.
} 


$$
\begin{aligned}
\frac{\partial \mathbf{U}^{(1)}}{\partial t} & +f \mathbf{k} \times \mathbf{U}^{(1)}+\left(H / H_{0}\right) \boldsymbol{\nabla} \eta^{(1)} \\
= & -\frac{\partial \mathbf{U}^{(0)}}{\partial \tau}-(\mathrm{Ro} / \epsilon)\left(\mathbf{U}^{(0)} \cdot \boldsymbol{\nabla}\right)\left(\mathbf{U}^{(0)} H_{0} / H\right)+\mathbf{F}(x, y, t, \tau)
\end{aligned}
$$

$$
\begin{aligned}
& \frac{\partial \eta^{(1)}}{\partial t}+\boldsymbol{\nabla} \cdot \mathbf{U}^{(1)} \\
& =-\frac{\partial \eta^{(0)}}{\partial \tau}-(\mathrm{Ro} / \epsilon) \boldsymbol{\nabla} \cdot\left(\eta^{(0)} \mathbf{U}^{(0)} H_{0} / H\right)+w_{p}(x, y, t, \tau)
\end{aligned}
$$

The boundary condition is that $\mathbf{U}^{(1)} \cdot \mathbf{n}=0$ away from the strait entrance, whereas $\mathbf{U}^{(1)} \cdot \mathbf{n}$ is specified by the strait formulation at the entrance.

The terms on the right-hand side of (3.2) depend on predetermined forcing and dissipation and on the lowest order solutions. Some of these terms may excite a resonant response in the $O(\epsilon)$ fields leading to secular growth of the solutions in $t$. Such behavior would eventually invalidate our asymptotic expansion, and the offending terms must be removed by restricting the slow time dependence of the lowest order solutions. [This procedure is discussed by Bender and Orszag (1978, chapter 11) in connection with ordinary differential equations, and our analysis is based on the same principles.] To do so, it is first necessary to identify the resonant terms using the property that such terms must project spatially and temporally onto the homogeneous solutions to (3.1) and (3.2). That is, a resonant term will have $\exp \left[i \omega_{k t}\right]$ time dependence, where $\omega_{k}$ is the frequency of any normal mode, and must have a spatial structure with nonzero projection onto that normal mode. Therefore, one may proceed by multiplying (3.1) and (3.2) by $\left(H_{0} / H\right) U_{j}^{*}$ and $\zeta_{j}^{*}$, respectively, and adding the products. [Recall that the latter are the complex conjugates of the lowest order horizontal structure functions and that the multiplication is the same procedure used to derive the orthogonality relationship (2.13).] Integrating the results over the area of the basin and applying the boundary condition (2.7) lead to

$$
\begin{aligned}
\left(\frac{\partial}{\partial t}-i \omega_{j}\right) \iint_{\mathcal{A}}\left[\frac{\mathbf{U}^{(1)} \cdot \mathbf{U}_{j}^{*}}{\left(H / H_{0}\right)}+\eta^{(1)} \zeta_{j}^{*}\right] d \sigma= & -\int_{0}^{W}\left[\zeta_{j}^{*} \mathbf{U}^{(1)} \cdot \mathbf{n}\right]_{e} d s-\iint_{\mathfrak{A}}\left[\frac{\mathbf{U}_{j}^{*} \cdot \partial \mathbf{U}^{(0)} / \partial \tau}{\left(H / H_{0}\right)}+\zeta_{j}^{*} \frac{\partial \eta^{(0)}}{\partial \tau}\right] d \sigma \\
& -\frac{\operatorname{Ro}}{\epsilon} \iint_{\mathcal{A}}\left[\left(\frac{H_{0}}{H}\right) \mathbf{U}_{j}^{*} \cdot\left(\mathbf{U}^{(0)} \cdot \boldsymbol{\nabla}\right)\left(\mathbf{U}^{(0)} H_{0} / H\right)+\xi_{j}^{*} \boldsymbol{\nabla} \cdot\left(\eta^{(0)} \mathbf{U}^{(0)} H_{0} / H\right)\right] d \sigma \\
& +\iint_{\mathscr{A}}\left(\mathbf{U}_{j}^{*} \cdot \mathbf{F} H_{0} / H+w_{p} \zeta_{j}^{*}\right) d \sigma
\end{aligned}
$$

The subscript $e$ denotes evaluation at the entrance of the strait and $\int_{0}^{W} d s$ represents integration across the dimensionless width $W$ of the entrance, clockwise around the basin edge.

Any terms on the right-hand side of (3.3) with $e^{i \omega_{j} t}$ time dependence will produce a solution growing algebraically in $t$. One such term is the second integral, which may be rewritten

$$
\begin{aligned}
& \frac{\partial}{\partial \tau} \iint_{\mathscr{A}}\left[\frac{\mathbf{U}_{j}^{*} \cdot \mathbf{U}^{(0)}}{\left(H / H_{0}\right)}+\zeta_{j}^{*} \eta^{(0)}\right] d \sigma \\
& =\frac{\partial A_{j}}{\partial \tau} e^{i \omega_{j} \mathrm{t}} \iint_{\mathcal{A}}\left[\frac{\mathbf{U}_{j}^{*} \cdot \mathbf{U}_{j}}{\left(H / H_{0}\right)}+\zeta_{j}^{*} \zeta_{j}\right] d \sigma
\end{aligned}
$$

by applying the orthogonality condition (2.13) to the expansion (2.8). The other integrals on the right-hand side of (3.3) may also have $e^{i \omega_{j} t}$ time dependence. To avoid resonance, all such terms must be removed, and it follows that

$$
\begin{aligned}
\frac{\partial A_{j}}{\partial \tau}=\{ & -\int_{0}^{W}\left[\zeta_{j}^{*} \mathbf{U}^{(1)} \cdot \mathbf{n}\right]_{e} d s-\frac{\mathrm{Ro}}{\epsilon} \iint_{\mathcal{A}}\left[\left(\frac{H_{0}}{H} \mathbf{U}_{j}^{*} \cdot \mathbf{U}^{(0)} \cdot \boldsymbol{\nabla}\right)\left(\mathbf{U}^{(0)} H_{0} / H\right)+\zeta_{j}^{*} \boldsymbol{\nabla} \cdot\left(\eta^{(0)} \mathbf{U}^{(0)} H_{0} / H\right)\right] d \sigma \\
& \left.+\iint_{\mathscr{A}}\left[\mathbf{U}_{j}^{*} \cdot \mathbf{F} H_{0} / H+w_{p} \zeta_{j}^{*}\right] d \sigma\right\} / \iint_{\mathfrak{A}}\left[\frac{\left|\mathbf{U}_{j}\right|^{2}}{\left(H / H_{0}\right)}+\left|\zeta_{j}\right|^{2}\right] d \sigma .
\end{aligned}
$$


The operator \{\}$_{j}$ selects the coefficients of any terms having $e^{i \omega_{j} t}$ time dependence in the bracket. The case $j$ $=0$ is excluded and will be considered separately in section 4. Note that (3.5) can be interpreted as an energy balance, with the right-hand side being a normalized sum of terms quantifying work done on the $j$ th mode by forcing $\mathbf{F}$, by the inflows and outflows $w_{e}$ and $\mathbf{U}^{(1)} \cdot \mathbf{n}$, and by other modes through the nonlinear interactions.

In evaluating the right-hand side of (3.5) $\zeta_{j}$ and $\mathbf{U}_{j}$ are given by the known, closed-basin modes and $\mathbf{F}$ and $w_{p}$ are prespecified or given in terms of $O(1)$ quantities. The normal component of the $O(\epsilon)$ entrance velocity $\mathbf{U}^{(1)} \cdot \mathbf{n}$ is supplied by the strait formulation. If multiple straits connect to the basin, (3.5) will contain a sum of entrance width integrals. When linear forms for $\mathbf{U}^{(1)} \cdot \mathbf{n}$, $\mathbf{F}$, and $w_{p}$ are used, the numerator in (3.5) will generally contain terms proportional to $A_{j}$. If $\omega_{j}$ is a multiple frequency, shared by modes $j$ and $k$ for instance, dependence on $A_{k}$ will also occur. ${ }^{4}$ Amplitudes of modes in different basins sharing the same frequency can also arise in the term $\left[\xi_{j}^{*} \mathbf{U}^{(1)} \cdot \mathbf{n}\right]_{e}$ if the strait flow is not hydraulically controlled. If $\left[\mathbf{U}^{(1)} \cdot \mathbf{n}\right]_{e}, F$, or $\omega_{e}$ are nonlinear in the $O(1)$ modes, the numerator of (3.5) may contain nonlinear dependencies on the amplitudes of modes having frequencies other than $\omega_{j}$. For hydraulically controlled outflow the dependence will be nonlinear unless further approximations are made. Finally, if $(\mathrm{Ro} / \epsilon)$ is $O(1)$, nonlinearities may arise from mode interactions in the basin interior [the middle term in the numerator of the right-hand side of (3.5)].

Platzman (1984) obtained a relation analogous to (3.5) in his treatment of World Ocean modes. Instead of a strait, he had a World Ocean continental shelf across which wave radiation could occur. His discussion involves a discretized model but the distinction is only superficial. In particular, his equation (12.9) is a discretized form of (3.5) in which the nonlinear interaction terms are absent, $w_{p}=F=0$, and where the strait entrance condition is replaced by a linear radiation condition.

\section{Evolution of the geostrophic "mode"}

In this section, evolution equations for the slow time dependence of the Helmholtz and geostrophic modes are derived. Unlike the oscillatory modes, each of which is identified with a discrete spatial structure, the geostrophic solutions are continuously distributed: any geostrophically balanced flow circulating around closed contours of constant $\mathrm{fH}_{0} / \mathrm{H}$ is a solution. Nevertheless,

\footnotetext{
${ }^{4}$ In studies using actual bathymetry (e.g., Platzman 1972; Candela and Lozano 1994), it is rare to find frequency multiplicity apart from $\omega=0$. In idealized geometries, however, multiplicity is quite common. For example, in a square basin with flat horizontal bottom and constant $f$, any mode having $x$ - and $y$-mode numbers $(n, m)$ will, by symmetry, share its frequency with the mode $(m, n)$
}

the procedure used to obtain the slow-time evolution of these solutions is similar to that for the oscillatory modes. As before, how the geostrophic modes are initialized must first be determined, given an arbitrary initial state. Doing so requires the development of a kind of orthogonality relation allowing the oscillatory modes to be eliminated from the initial state. The relation is obtained by performing a circulation integral around each closed geostrophic contour and showing that the circulation (or potential circulation) associated with the oscillatory modes is zero. Next, the evolution equation for the geostrophic mode is obtained. As before, terms leading to secular growth in $t$ of the $O(\epsilon)$ fields must be identified and suppressed, and this is done by performing circulation integrals of the $O(\epsilon)$ equations over each closed geostrophic contour. For the Helmholtz mode a similar set of operations is carried out using conservation of mass, rather than potential circulation, as the guiding constraint. [A reader who wishes to skip the technical details may proceed directly to the evolution equations (4.5) and (4.8) for the geostrophic and Helmholtz modes and the accompanying physical interpretations.] Finally, an alternate form of the geostrophic evolution equation, written in geostrophic coordinates and advantageous for numerical computations involving basins of complex topography, is given by (4.6).

The geostrophic and Helmholtz modes are contained in the fields denoted by $\eta_{g}(x, y, \tau)$ and $\mathbf{U}_{g}(x, y, \tau)$ and that $\tau$ dependence is not necessarily separable from the $(x, y)$ dependence. In the following, repeated use is made of the fact that $\eta_{g}$ is constant along geostrophic contours, as follows from (2.9) with $\omega_{j}=0$ :

$$
f \mathbf{k} \times \mathbf{U}_{g}=-\frac{H}{H_{0}} \nabla \eta_{g} .
$$

The geostrophic/Helmholtz solution is particularly important as it carries all excess mass, a property which can be shown by integrating (2.10) over the area of the basin and applying the boundary condition $\mathbf{U}_{k} \cdot \mathbf{n}=0$ on the periphery. The result

$$
\omega_{k} \iint_{\mathscr{A}} \zeta_{k} d \sigma=0
$$

shows that the net surface displacement for all but the steady $\left(\omega_{k}=0\right)$ modes is zero. The Helmholtz and geostrophic modes are therefore crucial to any problem involving exchange of mass between basin.

\section{a. Synthesis from initial data}

In the case where the upper boundary is rigid and the geostrophic contours are closed, Greenspan (1969) has shown that the geostrophic modes can be synthesized from arbitrary initial data by using the property that the oscillatory modes carry no net potential circulation. We can obtain a generalized result for a free upper boundary 
by first multiplying (2.5) by $H_{0} / H$, taking the curl of the result, and integrating over any area $\mathcal{A}_{c}$ bounded by a closed geostrophic contour $C$. (Geostrophic motion in regions of boundary-intersecting geostrophic contours is nil.) From this result one subtracts the area integral of (2.6) multiplied by $f H_{0} / H$ yielding

$$
\begin{aligned}
& \frac{\partial}{\partial t}\left[\iint_{\mathfrak{A}_{C}} \mathbf{k} \cdot \boldsymbol{\nabla} \times \frac{\mathbf{U}^{(0)} H_{0}}{H} d \sigma\right. \\
& \left.\quad-\left(f H_{0} / H\right)_{C} \iint_{\mathfrak{A}_{c}} \eta^{(0)} d \sigma\right]=0 .
\end{aligned}
$$

The modal version of this equation can be obtained by performing the same sequence of operations on (2.9) and (2.10), leading to

$$
\begin{aligned}
i \omega_{k}\left[\iint_{\mathfrak{A}_{c}} \mathbf{k} \cdot \boldsymbol{\nabla} \times\left(\mathbf{U}_{k} H_{0} / H\right) d \sigma\right. \\
\left.\quad-\left(f H_{0} / H\right)_{C} \iint_{\mathfrak{A}_{c}} \zeta_{k} d \sigma\right]=0 .
\end{aligned}
$$

This relation shows that the potential circulation (term in braces) about any geostrophic contour of any oscillatory $\left(\omega_{k} \neq 0\right)$ mode is zero.

The remaining procedure is discussed by Greenspan (1969) for circulation in a container with a rigid (though not necessarily horizontal) lid, in which case the free surface term is absent from (4.2). The generalization of his result is straightforward. To begin with, the geostrophic solution is projected onto the initial potential vorticity using (4.2a and $4.2 \mathrm{~b})$ :

$$
\begin{aligned}
& \iint_{\mathfrak{A}_{c}} \mathbf{k} \cdot \boldsymbol{\nabla} \times\left(\mathbf{U}_{g}(x, y, 0) H_{0} / H\right) d \sigma \\
& -\left(\frac{f H_{0}}{H}\right)_{C} \iint_{\mathfrak{A}_{c}} \eta_{g}(x, y, 0) d \sigma \\
& =\iint_{\mathcal{A}_{c}} \mathbf{k} \cdot \boldsymbol{\nabla} \times\left(\mathbf{U}^{(0)}(x, y, 0,0) H_{0} / H\right) d \sigma \\
& \quad-\left(f H_{0} / H\right)_{C} \iint_{\mathfrak{A}_{c}} \eta^{(0)}(x, y, 0,0) d \sigma .
\end{aligned}
$$

If $\mathrm{fH}_{0} / \mathrm{H}$ is nonuniform, the integral equation (4.3) must be solved for each area $\mathcal{A}_{c}$ in order to obtain the initial distribution of geostrophic velocity and surface elevation. In cases where the geometry as well as the initial total velocity and elevation possess certain symmetries, it may be straightforward to do so directly. In more complicated cases advantage can be taken of the fact that $\eta_{g}$ is constant along geostrophic contours and the corresponding functional dependence can be sought. The simplest case arises when all the closed geostrophic contours in the basin have unique $f H_{0} / H$ values so that $\eta_{g}\left(f H_{0} / H, 0\right)$ is single valued.

In general, the geostrophic contours will occur in a nest with the central contour $f H_{0} / H=\left(f H_{0} / H\right)_{0}$ enclosing zero area. Under these conditions (4.3) may be rewritten as

$$
\frac{d}{d \alpha}\left(\frac{J(\alpha)}{\alpha} \frac{d \eta_{g}(\alpha, 0)}{d \alpha}\right)-\mathcal{A}_{c}^{\prime}(\alpha) \eta_{g}(\alpha, 0)=(I(\alpha) / \alpha)^{\prime},
$$

where $\alpha$ represents $f H_{0} / H$ and $I(\alpha)$ and $J(\alpha)$ are given by (A.5) and (A.6) of the appendix. The steps leading to (4.4) are detailed in the appendix.

The two integration constants in the solution to the second-order equation equation (4.4) can normally be evaluated by a condition of boundedness at the central contour $\alpha_{0}$ and the condition that the solution contains all net initial surface displacement [implied by (4.1)].

If the basin contains multiple nests of closed contours, (4.4) must be solved separately in the different regions of monotonic $\mathrm{fH}_{0} / \mathrm{H}$ and the individual solutions must be matched.

\section{b. Compatibility condition for geostrophic solutions}

The $\tau$ dependence of the steady modes is obtained by performing the same sequence of operations on (3.1) and (3.2) that was used to derive (4.3). The result is the following integral differential equation

$$
\begin{aligned}
\frac{\partial}{\partial \tau}\left[\oint_{c} \frac{H_{0}}{H} \mathbf{U}_{g} \cdot \mathbf{l d} \mathbf{s}-\frac{f H_{0}}{H} \iint_{\mathfrak{A}_{c}} \eta_{g} d \sigma\right] \\
=\left\{\oint_{c} \frac{H_{0}}{H} \mathbf{F} \cdot \mathbf{l d} \mathbf{d}-\frac{f H_{0}}{H} \iint_{\mathfrak{A}_{c}} \omega_{p} d \sigma\right. \\
\left.\quad-\frac{R_{0}}{\epsilon} \oint_{c}\left(\mathbf{u}^{(0)} \cdot \mathbf{n}\right)\left[\frac{\partial}{\partial n} \mathbf{u}^{(0)} \cdot \mathbf{l}-\frac{f H_{0}}{H} \eta^{(0)}\right] d s\right\}_{0},
\end{aligned}
$$

where \{\}$_{0}$ selects only the $t$-independent terms, $\mathbf{u}^{(0)} \cdot \mathbf{n}$ is the cross-contour (ageostrophic) velocity, $\mathbf{u}^{(0)} \cdot \mathbf{l}$ is the clockwise positive along-contour velocity, $\partial\left(\mathbf{u}^{(0)} \cdot \mathbf{l}\right) / \partial n$ is the normal (outward) derivative of the latter, and $s$ measures distance positive clockwise around the contour. Equation (4.5) is a version of the circulation theorem stating that the slow change in potential circulation is associated with the net tangential force $\mathbf{F} \cdot \mathbf{d l}$ about the contour, the net entrainment velocity over the enclosed area, and the net inflow of relative vorticity and interface displacement by the cross-contour velocity $\mathbf{u}^{(0)} \cdot \mathbf{n}$. If the horizontal velocity is geostrophic, $\mathbf{u}^{(0)} \cdot \mathbf{u}_{g}$, then $\mathbf{u}^{(0)} \cdot \mathbf{n}$ vanishes and the terms multiplied by $\mathrm{Ro} / \epsilon$ disappear. Thus, the interior nonlinear interaction terms vanish for geostrophic flow in the presence of continuously varying $\mathrm{fH}_{0} / \mathrm{H}$ contours. Nonlinearities may still result from the boundary conditions, as shown below, or from non- 
linear dependencies in the forcing function $\mathbf{F}$. If $\mathrm{fH}_{0} / \mathrm{H}$ is uniform within a finite area so that all closed contours have constant $f H_{0} / H, \mathbf{u}_{g} \cdot \mathbf{n}$ need no longer vanish and nonlinear interactions occur. This situation arises in the quasigeostrophic limit of the strait model as shown in section 4c.

An alternative form of (4.5) may be obtained by expressing $\eta_{g}$ as a function of $f H_{0} / H$ (or $\alpha$ ) and assuming single valuedness as before. To do so, integrate (4.5) with respect to $\tau$, apply Eqs. (A.1) and (A.2), divide by $\alpha$, and differentiate the result with respect to $\alpha$, leading to

$$
\begin{aligned}
& \frac{\partial}{\partial \alpha}\left(\frac{J(\alpha)}{\alpha} \frac{\partial \eta_{g}}{\partial \alpha}\right)-\frac{d \mathcal{A}_{c}}{d \alpha} \eta_{g} \\
& \quad=\frac{\partial}{\partial \alpha}\left(\frac{1}{\alpha} \int_{0}^{\tau} K\left(\alpha, \tau^{\prime}\right) d \tau^{\prime}\right)+d(I(\alpha) / \alpha) d \alpha,
\end{aligned}
$$

where

$$
\begin{aligned}
K\left(\alpha, \tau^{\prime}\right) & \\
= & \left\{\iint_{\mathcal{A}_{c}} \mathbf{k} \cdot \boldsymbol{\nabla}\right. \\
& \times\left[\frac{\mathbf{F} H_{0}}{H}-\frac{\operatorname{Ro} H_{0}}{\epsilon H}\left(\mathbf{U}^{(0)} \cdot \boldsymbol{\nabla}\right)\left(\mathbf{U}^{(0)} H_{0} / H\right)\right] d \sigma \\
& \left.\left.-\alpha \iint_{\mathscr{A}_{c}}\left[w_{p}-\frac{\mathrm{Ro}}{\epsilon} \boldsymbol{\nabla} \cdot\left(\eta^{(0)} \mathbf{U}^{(0)}\right) H_{0} / H\right)\right] d \sigma\right\}_{0} .
\end{aligned}
$$

Our experience with simple analytical examples has been that it is easier to use the more primitive form (4.5) to solve for $\eta_{g}$ and that (4.6) proves more advantageous in complicated examples requiring numerical algorithms. In either case, two boundary conditions need to be specified and one will generally continue to be provided by a requirement of boundedness at the central contour. The second condition can be obtained by integrating (3.2) over the area $\mathcal{A}$ of the basin. After applying boundary conditions, using (4.1), and suppressing secular terms, one obtains

$$
\frac{\partial}{\partial \tau} \iint_{\mathscr{R}} \eta_{g} d \sigma=\left\{\iint_{\mathscr{R}} w_{p} d \sigma-\int_{0}^{W} \mathbf{U}^{(1)} \cdot \mathbf{n} d s\right\}_{0},
$$

which is nothing more than a mass budget. An alternative condition, which is convenient to apply when a velocity component (rather than $\eta_{g}$ ) is the dependent variable, can be derived by applying (4.5) at the outer contour corresponding to the basin edge (i.e., set $\mathcal{A}_{c}=$ $\mathcal{A})$. After using Green's theorem and the divergence theorem, applying boundary conditions, and using (4.8),

$$
\begin{aligned}
& \frac{\partial}{\partial \tau} \oint_{C_{e}}\left(\frac{H_{0}}{H}\right) \mathbf{U}_{g} \cdot \mathbf{l} d s \\
& \quad=\oint_{C_{e}} \frac{H_{0}}{H} \mathbf{F} \cdot \mathbf{l} d s-\frac{f H_{0}}{H} \int_{0}^{W} \mathbf{U}^{(1)} \cdot \mathbf{n} d s,
\end{aligned}
$$

where $C_{e}$ denotes the basin edge (including the strait entrance) and $\mathbf{I}$ is a unit tangent to $C_{e}$. Equation (4.9) is a form of the circulation theorem for the basin as a whole. If the basin contains boundary-intersecting geostrophic contours, one simply sets $\mathbf{U}_{g}=0$ along such contours. If the boundary itself is not a geostrophic contour, (4.8) continues to hold, or (4.9) can be applied by considering $C_{e}$ to be the outermost closed geostrophic contour. If $H$ is zero along $C_{e}$, as will occur in all cases where the side walls are nonvertical, $\left(H_{0} / H\right) \mathbf{U}_{g}$ should simply be thought of as $\mathbf{u}_{g}$ (the geostrophic velocity) and (4.5) and (4.9) should be reformulated accordingly.

A more difficult circumstance arises when several nests of geostrophic contours are present, for then $\eta_{g}$ is no longer a single-valued function of $\mathrm{fH}_{0} / \mathrm{H}$. In such cases, (4.5) or (4.6) must be solved separately in the individual nests and their $\eta_{g}$ values matched at common boundaries.

\section{c. Reduction to quasigeostrophic theory}

Finally, it is easy to deduce $f$-plane quasigeostrophic theory from (4.5). First, one must pose geostrophic initial conditions so that no oscillatory modes are excited, and $U^{(0)}$ and $\eta^{(0)}$ may be replaced by $U_{g}$ and $\eta_{g}$ on the right-hand side. Next, one assumes that $f$ and $H$ are uniform ( $f=1$ and $\left.H=H_{0}\right)$ over the entire basin so that any closed contour is a constant $f H_{0} / H$ contour. Under this condition all integrands in (4.5) must balance pointwise throughout the basin, leading to

$$
\left(\frac{\partial}{\partial \tau}+\frac{\operatorname{Ro}}{\epsilon} \mathbf{u}_{g} \cdot \boldsymbol{\nabla}\right)\left(\frac{\partial v_{g}}{\partial x}-\frac{\partial u_{g}}{\partial y}-\eta_{g}\right)=\mathbf{k} \cdot \boldsymbol{\nabla} \times \mathbf{F}-w_{p},
$$

the $f$-plane, quasigeostrophic potential vorticity equation.

\section{Treatments of straits}

We now give examples of how some of the more widely used laws for strait and sill flows can be adapted for use in the asymptotic theory. For self-consistency it must be possible to configure the strait such that the normal component of entrance transport velocity is nondimensionally small:

$$
\frac{\tilde{\mathbf{U}}_{e} \cdot \mathbf{n}}{\left(g H_{0}\right)^{1 / 2} \mathcal{N}}=\epsilon \mathbf{U}_{e}^{(1)} \cdot \mathbf{n} \ll 1,
$$

where the definition of $\epsilon$ depends on the strait model. Furthermore, the $O(\epsilon)$ transport velocity $\tilde{\mathbf{U}}_{e}^{(1)} \cdot \mathbf{n}$ must be expressible in terms of known quantities such as $O(1)$ fields or prescribed forcing, or in terms of quantities 
that can be calculated from other compatibility relations in neighboring basins. Most of our attention will be devoted to the adaption of a rotating hydraulic model due to Whitehead et al. (1974, hereafter WLK). Later we consider strait formulations based on theories for frictionally or geostrophically controlled throughflow.

In all the cases discussed below, the along-strait velocity component is assumed to be in geostrophic balance so that the transport scales with the difference in the square of the surface elevation across the strait. An $O(1)$ variation of $\eta^{(0)}$ across the strait entrance therefore implies $O(1)$ transport velocity at the strait entrance in violation of the underlying assumption $\mathbf{U}_{e} \cdot d \mathbf{n}=O(\epsilon)$. Use of geostrophic strait formulations therefore requires that we restrict attention to situations in which $\eta^{(0)}$ is constant (or nearly so) across the entrance. If the basin flow is geostrophic and the entrance lies along a geostrophic contour, then the interior dynamics ensure that $\eta^{(0)}$ is constant, as desired. If the entrance does not lie along a geostrophic contour, it can often be redefined to do so. For time-dependent interior flows, the entrance width $W$ must be assumed much smaller than the typical scale of variation of $\eta^{(0)}$, eliminating modes of fine cross-entrance horizontal structure from consideration. In all cases the horizontal structure function $\zeta_{j}(x, y)$ must be nearly constant across the entrance, and the term

$$
\int_{0}^{W}\left[\zeta_{j}^{*} \mathbf{U}^{(1)} \cdot d \mathbf{n}\right]_{e} d s
$$

appearing in (3.5) may therefore be approximated by

$$
\left[\zeta_{j}^{*}\right]_{e} \int_{0}^{W} \mathbf{U}_{e}^{(1)} \cdot \mathbf{n} d s,
$$

where $\left[\zeta_{j}^{*}\right]_{e}$ is the value of $\zeta_{j}^{*}$ evaluated at any convenient location across the entrance. With this simplification evaluation of (3.5) requires specification of the total outflow transport relation but not information about the entrance velocity distribution. The only other place where the outflow condition enters the formulation is (4.8) [or its alternate form (4.9)], where, again, the total transport relation is required. Hence, determination of the lowest order basin flow requires that one know only the total transport relation and not the actual velocity distribution.

\section{a. Inviscid, hydraulically controlled outflows according to WLK}

The basic result due to WLK can be described as follows. Referring to Fig. 2a, consider a uniformly rotating flow in a channel of rectangular cross section that spills over a sill. The fluid thickness at the sill crest is assumed to be much less than the upstream thickness so that severe vortex squashing is implied for the fluid as it approaches the sill. Under these conditions, hydraulic control theory determines the total dimensional volume transport as

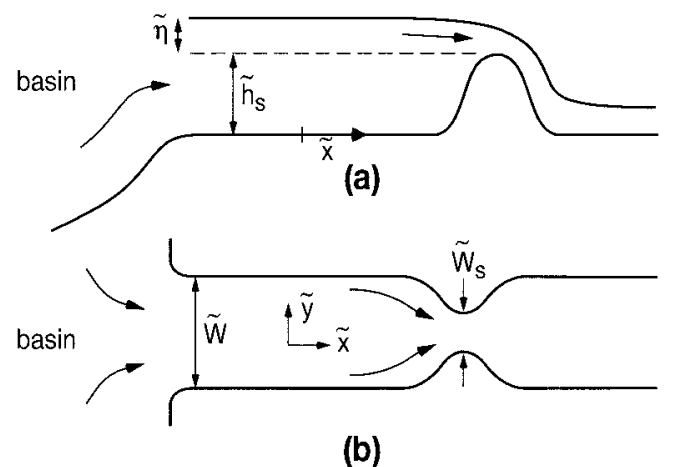

FIG. 2. Definition sketch showing strait with rectangular section exiting from basin.

$$
\begin{gathered}
\tilde{T}=\left(\frac{2}{3}\right)^{3 / 2} \tilde{W}_{s} g^{1 / 2}\left[\tilde{\eta}_{r}-\frac{f_{0}^{2} \tilde{W}_{s}^{2}}{8 g}\right]^{3 / 2} \\
{\left[\text { if } \tilde{W}_{s}<\left(2 g^{\prime} \tilde{\eta}_{r} / f_{0}^{2}\right)^{1 / 2}\right],}
\end{gathered}
$$

where $\tilde{W}_{s}$ is the channel width at the sill and $\tilde{\eta}_{r}$ is the elevation of the free surface above the sill measured along the right-hand wall (facing downstream) as indicated in Fig. 2b. [In adapting (5.1a) to our model, $\tilde{\eta}_{r}$ may be measured at any location across the entrance.] In Eq. (5.1a) the layer thickness is assumed nonzero across the entire sill width, which holds as long as $\tilde{W}_{s}$ $<\left(2 g^{\prime} \tilde{\eta}_{r} / f_{0}^{2}\right)^{1 / 2}$. If the latter condition fails, implying that the left-hand edge of the current has separated from the side wall, (5.1a) is replaced by

$$
\tilde{T}=\frac{g^{\prime} \tilde{\eta}_{r}^{2}}{2 f_{0}} \quad\left[\text { if } \tilde{W}_{s}>\left(2 g^{\prime} \tilde{\eta}_{r} / f_{0}^{2}\right)^{1 / 2}\right] .
$$

There is some question as to whether separated sill flow can actually occur under conditions of hydraulic control with rectangular geometry [see Stern (1980) and Pratt (1987) for evidence to the contrary], and caution should be exercised in using (5.1b).

We now assume that the length of the strait is sufficiently small that the flow therein may be considered quasi-steady. It is also assumed that the strait geometry varies gradually in the alongaxis direction so that the streamlines at any cross section are nearly parallel. Near the strait entrance, the scales characterizing the basin flow should continue to hold, and thus the dimensionless transport corresponding to $(5.1 \mathrm{a})$ or $(5.1 \mathrm{~b})$ is

$$
\frac{\tilde{T}}{\operatorname{Ro}\left(g H_{0}\right)^{1 / 2} H_{0} L_{d}}=\left\{\begin{array}{c}
\left(\frac{2}{3}\right)^{3 / 2} \operatorname{Ro}^{1 / 2}\left(\frac{\tilde{W}_{s}}{L_{d}}\right)\left[\eta_{r}-\frac{f_{0}^{2} \tilde{W}_{s}^{2}}{8 g N}\right]^{3 / 2} \\
\left.\frac{\tilde{W}_{s}}{L_{d}}<(2 \mathrm{Ro})^{1 / 2} \eta_{r}^{1 / 2}\right) \\
\frac{1}{2} \operatorname{Ro} \eta_{r}^{2}, \\
\left.\frac{\tilde{W}_{s}}{L_{d}} \geq(2 \mathrm{Ro})^{1 / 2} \eta_{r}^{1 / 2}\right) .
\end{array}\right.
$$


In either case the dimensionless transport is proportional to a small parameter ( $\mathrm{Ro}$ or $\left.\mathrm{Ro}^{1 / 2}\right)$, consistent with the basic assumption of weak outflow. The requirement of (5.2a) can only be satisfied if $\tilde{W}_{s} / L_{d}$ is $O\left(\mathrm{Ro}^{1 / 2}\right)$ so that the dimensionless transport is actually $O(\mathrm{Ro})$ in either case.

In summary, the small parameter $\epsilon$ characterizing the strength of the outflow is Ro. Nonlinear self-interaction terms arise in (3.5) at the same order of approximation as the outflow term. The latter is given by

$$
\begin{aligned}
& \int_{0}^{W} \zeta_{j}^{*} \mathbf{U}^{(1)} \cdot \mathbf{n} d s \\
& =\left[\zeta_{j}^{*}\right]_{e}\left\{\begin{array}{c}
\left(\frac{2}{3}\right)^{3 / 2}\left(\frac{W_{s}}{\mathrm{Ro}^{1 / 2}}\right)\left[\eta_{r}^{(0)}-\frac{W_{s}^{2}}{8 \mathrm{Ro}}\right]^{3 / 2} \\
W_{s}<(2 \mathrm{Ro})^{1 / 2} \eta_{\tau}^{(0) 1 / 2} \\
\frac{1}{2} \eta_{r}^{(0) 2}, \\
W_{s}>(2 \mathrm{Ro})^{1 / 2} \eta_{r}^{(0) 1 / 2},
\end{array}\right.
\end{aligned}
$$

where $W_{s}=\tilde{W}_{s} / L_{d}$ and $W_{s} / \operatorname{Ro}^{1 / 2}$ is understood to be $O(1)$. In the limit of weak rotation (or vanishing strait width), $W_{s} / \mathrm{Ro}^{1 / 2}=\tilde{W}_{s} f_{0} /(g \mathcal{N})^{1 / 2} \rightarrow 0$ and the above expression can be approximated by

$$
\begin{gathered}
\int_{0}^{W} \zeta_{j}^{*} \mathbf{U}^{(1)} \cdot \mathbf{n} d s=\left[\zeta_{j}^{*}\right]_{e}\left(\frac{2}{3}\right)^{3 / 2}\left(\frac{W_{s}}{\mathrm{Ro}^{1 / 2}}\right) \eta_{r}^{(0) 3 / 2}, \\
\left(\frac{\tilde{W}_{s} f_{0}}{(g \mathcal{N})^{1 / 2}} \rightarrow 0\right) .
\end{gathered}
$$

\section{b. Frictionally dominated outflows}

When friction is significant in the strait, a spatially averaged version of the alongstrait momentum equation is often used as a model. A typical example (Candela and Lozano 1994) is (in dimensional terms)

$$
\frac{\partial \tilde{U}_{s}}{\partial \tilde{t}}=-\frac{g H_{s}}{L_{s}}\left(\Delta \tilde{\eta}_{e}-\Delta \tilde{\eta}_{\alpha}\right)-R_{f} \tilde{U}_{s}
$$

where $\tilde{U}_{s}$ and $H_{s}$ are the width-averaged transport velocity and depth, both assumed uniform over the length $L_{s}$ of the strait. A linear drag law with coefficient $R_{f}$ is assumed and $\Delta \tilde{\eta}_{e}$ and $\Delta \tilde{\eta}_{\alpha}$ are the difference in actual and isostatic surface levels between the two ends of the strait.

Nondimensionalizing (5.5) leads to

$$
\begin{aligned}
\epsilon \mathbf{U}_{e} \cdot \mathbf{n} & =\frac{\tilde{U}_{s}}{\left(g H_{0}\right)^{1 / 2} \mathcal{N}} \\
& =\frac{-\left(g H_{0}\right)^{1 / 2}}{R_{f} L_{s}} \frac{H_{s}}{H_{0}}\left[\left(\Delta \eta_{e}-\Delta \eta_{\alpha}\right)+\frac{f_{0} L_{s}}{\left(g H_{0}\right)^{1 / 2}} \frac{\partial u_{s}}{\partial t}\right],
\end{aligned}
$$

where $H_{s}(y)$ is the strait depth, usually assumed to be constant and $u_{s}=U_{s} H_{0} / H_{s}$ is the nondimensional strait velocity. For $L_{s} \leq\left(g H_{0}\right)^{1 / 2} / f_{0}$, the entrance transport velocity is constrained by the parameter

$$
\epsilon=\frac{\left(g H_{0}\right)^{1 / 2}}{R_{f} L_{s}} \frac{H_{s}}{H_{0}},
$$

which is small for sufficiently large $R_{f}$. For $\epsilon \ll 1$ and $f_{0} L_{s} /\left(g H_{0}\right)^{1 / 2} \ll 1$, the total transport is approximated by

$$
\int_{0}^{W} \mathbf{U}_{e} \cdot \mathbf{n} d y=-\left(\Delta \eta_{e}-\Delta \eta_{\alpha}\right) W,
$$

where $W$ is the dimensionless strait width, assumed constant.

\section{c. Inviscid, geostrophically controlled outflows}

Geostrophic control (Garrett and Toulany 1982) is based on the premise that the cross-strait change in surface or interface displacement cannot be greater than $\Delta \eta$. Since the cross-strait change in $\eta$ determines the geostrophic transport, the result is a transport bound whose value can be obtained by defining

$$
\epsilon=\frac{\left(g H_{0}\right)^{1 / 2}}{W f_{0}} \frac{H_{s}}{H_{0}}
$$

and using (5.7). Models of fluctuating flow in straits have suggested that geostrophic control is valid only within certain frequency ranges (Pratt 1991; Hannah 1992) and under certain geometries (Wright 1987).

The models for frictionally and geostrophically controlled flow both imply coupling with the downstream basin. As opposed to the situation with regard to singlelayer hydraulic control, reversals of total transport are permitted.

The results of this section are summarized in Table 2.

\section{Discussion}

We believe that the compatibility conditions developed herein [(3.5), (4.5), (4.8), or the alternate forms (4.6) and (4.9)] could form the basis for investigations of a wide variety of physical processes occurring in semienclosed seas or basins. The underlying equations can be viewed as an extension of quasigeostrophic theory in which the basic (geostrophic) state has been generalized to include natural basin modes. Applications include steady circulations set up by basin flow fed from above and subject to dissipation (see Part II) as well as 
TABLE 2. Examples of strait models.

\begin{tabular}{|c|c|c|c|}
\hline Setting* & $\int \oint W \mathbf{U}_{\mathrm{e}}^{(1)} \cdot \mathrm{n} d s$ & & $\begin{array}{c}\text { Small } \\
\text { parameter, }\end{array}$ \\
\hline $\begin{array}{l}\text { 1. Nonrotating, } 1 \mathrm{D} \text {, hydraulically } \\
\text { controlled }\end{array}$ & $\begin{array}{l}(2 / 3)^{3 / 2}\left(W_{s} / \mathrm{Ro}^{1 / 2}\right) \eta_{r}^{3 / 2} \\
0\end{array}$ & $\begin{array}{l}\left(\eta_{r}^{(0)}>0\right) \\
\left(\eta_{r}^{(0)} \leq 0\right)\end{array}$ & $\operatorname{Ro}\left(=\frac{N}{H_{0}}\right)$ \\
\hline $\begin{array}{l}\text { 2. Rotating, hydraulically controlled, } \\
\text { uniform potential vorticity }\end{array}$ & $\begin{array}{l}(2 / 3)^{3 / 2}\left(W_{s} / \operatorname{Ro}^{1 / 2}\right)\left[\eta_{r}^{(0)}-\frac{W_{s}^{2}}{8 \mathrm{Ro}}\right]^{3 / 2} \\
1 / 2\left(\eta_{r}^{(0)}\right)^{2}\end{array}$ & $\begin{array}{l}\left(\left[(2 \mathrm{Ro})^{1 / 2}\left(\eta_{r}^{(0)}\right)^{1 / 2}\right]>W_{s}\right. \\
\left(\left[(2 \mathrm{Ro})^{1 / 2}\left(\eta_{r}^{(0)}\right)^{1 / 2}\right]<W_{s},\right. \\
\left.\quad \eta_{r}^{(0)}>0\right)\end{array}$ & $\operatorname{Ro}\left(=\frac{N}{H_{0}}\right)$ \\
\hline & 0 & $\left(\eta_{r}^{(0)} \leq 0\right)$ & $\operatorname{Ro}\left(=\frac{N}{H_{0}}\right)$ \\
\hline 3. Geostrophic control & $\left(\Delta \eta_{e}^{(0)}-\Delta \eta_{\alpha}\right) W$ & & $\frac{\left({ }_{g} H_{0}\right)^{1 / 2}}{W f_{0}} \frac{H_{s}}{H_{0}}$ \\
\hline 4. Frictionally dominated & $\left(\Delta \eta_{e}^{(0)}-\Delta \eta_{\alpha}\right) W$ & & $\frac{\left(g H_{0}\right)^{1 / 2}}{R L_{s}} \frac{H_{s}}{H_{0}}$ \\
\hline
\end{tabular}

* Cross sections are generally assumed rectangular. Other restrictions may apply, as described in the text.

forced and free oscillations in the presence of a hydraulic control (Part III). Although calculations are easiest when the horizontal structure functions of the modes are known analytically, as will be the case in certain idealized geometries, the use of complicated geometry or topography presents no intrinsic restriction; the normal modes are found numerically and the compatibility equations are simply formulated with numerically determined coefficients. Finally, applications are not limited to problems involving straits and sills. Our evolution equations could also be used to explore resonant interactions between Kelvin and Rossby modes and other issues involving nonlinear circulations in closed basins.

Having said this, we should also point out that there are many types of problems where our theory will be invalid or will fail to provide any fundamental simplification. First of all, the normal mode representation will generally not be useful for highly localized features such as isolated eddies set up by individual convection chimneys. Such features do not feel the basin geometry strongly and the high normal modes needed to synthesize them are less likely to conform to the parameter restrictions required by most outflow models. The largescale accumulation of mass by a group of chimneys would be a more suitable forcing mechanism. If the forcing, dissipation, or inflows and outflow are nondimensionally strong our method can only hope to provide intuition into certain dynamical aspects. Also, situations may arise in which the $O(1)$ solutions are identically zero and one must calculate the $O(\epsilon)$ solutions to capture the leading physical effects. An example would be a case with periodic and zero-mean forcing at a nonresonant frequency $\left(\neq \omega_{j}\right.$ for any $j$ ) and with no modes initially present. The lowest order finite response would be contained in the $O(\epsilon)$ fields and would have to be calculated directly from Eqs. (3.1) and (3.2). We note, however, that the dense packing of mode frequencies about $\omega=\mathrm{O}$ means that any subinertial forcing will be either resonant or near-resonant. In summary, the method would seem best suited for situations in which the forcing is subinertial and affects a significant fraction of basin area.

Even in cases where the $O(1)$ solution is nonzero, it may be desirable to compute certain aspects of the $O(\epsilon)$ solution, depending on what type of information is desired. In Part II, for example, we find that calculation of parcel trajectories from the basin interior to the strait entrance requires some consideration of the $O(\epsilon)$ fields near the boundaries.

Although other rotating hydraulic models exist (e.g., Gill 1977; Dalziel 1991; Killworth 1992), the WLK model is the only one that is both consistent with simplified dynamics assumed in the basin and does not predetermine the potential vorticity distribution of the escaping fluid. (The WLK model is not restricted to zero potential vorticity as sometimes thought.) This model requires the sill height to be nearly as large as the layer thickness near the entrance. If the sill is not high, and the choking mechanism is instead provided by a width contraction, another model must be used. Gill's (1977) work is applicable in this case but is restricted to uniform potential vorticity. This state of affairs highlights the need for development of general hydraulic laws which, in particular, can provide an entrance condition for arbitrary potential vorticity distributions.

Acknowledgments. This work was supported by the National Science Foundation under Grant OCE9115359. The authors wish to thank Joeseph Pedlosky, George Platzman, and Karin Borenäs for helpful suggestions. We also thank Deborah Taylor for preparing the manuscript. 
APPENDIX A

\section{Derivation of (4.4)}

Start by rewriting the integrals in (4.3) as follows:

$$
\begin{aligned}
& \iint_{\mathfrak{A}_{c}} \mathbf{k} \cdot \boldsymbol{\nabla} \times\left(\frac{\mathbf{U}_{g} H_{0}}{H}\right) d \sigma \\
& =\oint_{c} \frac{\mathbf{U}_{g} H_{0}}{H} \cdot d \mathbf{l}=\oint_{c} \frac{1}{f} \boldsymbol{\nabla} \eta_{g} \cdot d \mathbf{n} \\
& =\oint_{c} \frac{1}{f} \frac{\partial \eta_{g}}{\partial\left(f H_{0} / H\right)} \boldsymbol{\nabla}\left(\frac{f H_{0}}{H}\right) \cdot d \mathbf{n} \\
& =\frac{\partial \eta_{g}}{\partial\left(f H_{0} / H\right)} \oint_{c} \frac{1}{f} \nabla\left(\frac{f H_{0}}{H}\right) \cdot d \mathbf{n}
\end{aligned}
$$

and

$$
\begin{aligned}
& \frac{f H_{0}}{H} \iint_{\mathcal{A}_{c}} \eta_{g} d \sigma \\
& \quad=\frac{f H_{0}}{H} \int_{\left(f H_{0} / H\right)_{0}}^{\left(f H_{0} / H\right)} \eta_{g} \frac{d \mathcal{A}_{c}}{d\left(f H_{0} / H\right)} d\left(f H_{0} / H\right),
\end{aligned}
$$

where $\mathbf{n}$ and $\mathbf{l}$ represent outward normal and tangential unit vectors to the contour $C$ and all expressions are evaluated at $\tau=0$. Substituting these expressions into (4.3) leads to

$$
\frac{d \eta_{g}(\alpha, 0)}{d \alpha} J(\alpha)-\alpha \int_{\alpha_{0}}^{\alpha} \frac{d \mathcal{A}_{c}}{d \tilde{\alpha}} \eta_{g} d \tilde{\alpha}=I(\alpha),
$$

where $\alpha$ represents $f H_{0} / H$,

$$
J(\alpha)=\int_{c} \frac{1}{f} \nabla \alpha \cdot d \mathbf{n}
$$

and

$$
\begin{aligned}
I(\alpha)= & \iint_{\mathfrak{A}_{c}} \mathbf{k} \cdot \boldsymbol{\nabla} \times\left(\frac{\mathbf{U}^{(0)}(x, y, 0,0) H_{0}}{H}\right) d \sigma \\
& -\left(\frac{f H_{0}}{H}\right)_{C} \iint_{\mathfrak{A}_{c}} \eta^{(0)}(x, y, 0,0) d \sigma .
\end{aligned}
$$

In the rigid-lid case considered by Greenspan (1969), the free surface term (A.2) is absent and (A.3) reduces to

$$
\frac{d \eta_{g}(\alpha, 0)}{d \alpha}=\frac{I(\alpha)}{J(\alpha)},
$$

where $\eta_{g}$ should be interpreted as a pressure. In the more general case, differentiation of (A.3)/ $\alpha$ yields (4.4).

\section{REFERENCES}

Baines, P. G., 1995: Topographic Effects in Stratified Flows. Cambridge University Press, $482 \mathrm{pp}$.

Bender, C. M., and S. A. Orszag, 1978: Advanced Mathematical Methods for Scientists and Engineers. McGraw-Hill, 593 pp.

Bryden, H., and T. Kinder, 1991: Recent developments in strait dynamics. Rev. Geophys., (Suppl.), 617-631.

Candela, J., and C. J. Lozano, 1994: Barotropic response of the Western Mediterranean to observed atmospheric pressure forcing. Submitted to The Seasonal and Interannual Variability of the Western Mediterranean Sea, P. E. Violette, Ed., Coastal and Estuarine Studies, Vol. 46, Amer. Geophys. Union, 325-359.

Csanady, G. T., 1978: The arrested topographic wave. J. Phys. Oceanogr., 8, 47-62.

Dalziel, S. B., 1991: Two-layer hydraulics: A functional approach. J. Fluid Mech., 223, 135-163.

Dickson, R. R., E. M. Gmitrowicz, and A. J. Watson, 1990: Deepwater renewal in the northern North Atlantic. Nature, 334, 848850.

Garrett, C. J. R., 1975: Tides in gulfs. Deep-Sea Res., 22, 23-35.

$\longrightarrow$, and B. Toulany, 1982: Sea level variability due to meteorological forcing in the northeast Gulf of St. Lawrence. J. Geophys. Res., 87, 1968-1978.

Gill, A. E., 1977: The hydraulics of rotating-channel flow. J. Fluid Mech., 80, 641-671.

Greenspan, H. P., 1969: The Theory of Rotating Fluids. Cambridge University Press, $328 \mathrm{pp}$.

Hannah, C. G., 1992: Geostrophic control with wind forcing: Application to Bass Strait. J. Phys. Oceanogr., 22, 1596-1599.

Killworth, P. D., 1992: Flow properties in rotating, stratified hydraulics. J. Phys. Oceanogr., 22, 997-1017.

— 1994: On reduced-gravity flow through sills. Geophys. Astrophys. Fluid Dyn., 75, 91-106.

Miles, J. W., 1971: Resonant response of harbors: An equivalentcircuit analysis. J. Fluid Mech., 46, 241-266.

Platzman, G. W., 1972: Two-dimensional free oscillations in natural basins. J. Phys. Oceanogr., 2, 117-138.

— 1 1984: Normal modes of the World Ocean. Part III: A procedure for tidal synthesis. J. Phys. Oceanogr., 14, 1521-1531.

Pratt, L. J., 1987: Rotating shocks in a separated laboratory channel flow. J. Phys. Oceanogr. 17, 483-491.

— Ed., 1990: The Physical Oceanography of Sea Straits. Kluwer, $587 \mathrm{pp}$.

— 1991: Geostrophic versus critical control in straits. J. Phys. Oceanogr., 21, 728-732.

— 1997: Hydraulically drained flows in rotating basins. Part II: Steady flow. J. Phys. Oceanogr., 27, 2522-2535.

— flow. Annu. Rev. Fluid Mech., 23, 81-106.

Stern, L. J., 1980: Geostrophic fronts, bores, breaking and blocking waves. J. Fluid Mech., 99, 687-703.

Veltkamp, G. H., 1960: Spectral properties of Hilbert space operators associated with tidal motions. Ph.D. thesis, University of Utrecht, $91 \mathrm{pp}$.

Whitehead, J. A., A. Leetma, and R. A. Knox, 1974: Rotating hydraulics of strait and sill flows. Geophys. Fluid Dyn., 6, 101125.

Wright, D. G., 1987: Comments on "Geostrophic control of fluctuating barotropic flow through straits." J. Phys. Oceanogr., 17, 2375-2377. 